\title{
Difference Sphere: An Approach to Near Light Source Estimation
}

\author{
Takeshi Takai Koichiro Niinuma ${ }^{\dagger} \quad$ Atsuto Maki Takashi Matsuyama \\ Graduate School of Informatics, Kyoto University \\ Yoshida-Honmachi, Sakyo-ku, Kyoto 606-8501 Japan \\ \{takesi-t, niinuma, maki, tm\}@ vision.kuee.kyoto-u.ac.jp
}

\begin{abstract}
We present a novel approach for estimating lighting sources from a single image of a scene that is illuminated by near point light sources, directional light sources and ambient light. We propose to employ a pair of reference spheres as light probes and introduce the difference sphere that we acquire by differencing the intensities of two image regions of the reference spheres. Since the effect by directional light sources and ambient light is eliminated by differencing, the key advantage of considering the difference sphere is that it enables us to estimate near point light sources including their radiance, which has been difficult to achieve in previous efforts where only distant directional light sources were assumed. We also show that analysis of gray level contours on spherical surfaces facilitates separate identification of multiple combined light sources and is well suited to the difference sphere. Once we estimate point light sources with the difference sphere, we update the input image by eliminating their influence and then estimate other remaining light sources, that is, directional light sources and ambient light. We demonstrate the effectiveness of the entire algorithm with experimental results.
\end{abstract}

\section{Introduction}

Acquiring the knowledge of light sources is crucial in computer vision as well as in computer graphics especially with the recent advent of image based rendering techniques. Once the parameters of light sources are obtained, the illumination information can be effectively utilized for manipulating shadows, highlights, or shading on real/virtual object in images. In this paper, we consider the problem of estimating several co-existing light sources from a single image using a pair of spheres whose surface has Lambertian property. In particular, we deal with near point light sources, besides directional light source and ambient light, so that the reality of manipulated images should be increased.

The problem of estimating illuminant directions arises in the context of shape from shading [1] and early work focused on recovering a single distant light source assuming Lambertian surface and uniform albedo of target object. See

$\dagger$ Currently at Peripheral Systems Laboratories, Fujitsu Laboratories Limited.niinuma.k@jp.fujitsu.com
$[2,3]$ for a few example. Since, there have been successful attempts to recover a more general illumination description [4]. Marschner and Greenberg [5] propose a method for estimating directions and radiant intensities of a few light sources in a scene by a least-squares method for surface intensity functions. In [6], Yang and Yuille also solve for a small number of light sources by exploiting the constraints by occluding boundary. As an extension, Zhang and Yang [7] introduce a technique for estimating directions and radiant intensities of multiple light sources. While they analyze a single image of a reference sphere with known shape and Lambertian reflectance model, Zhou and Kambhamettu [8] extract these properties using stereo images of a reference sphere with specular reflection. They estimate the directions by the positions of highlights and radiant intensities from shading. Also, Maki [9] utilizes multiple images of an object that is in motion for estimating the illuminant direction by observing the brightness variance derived on the object surface. However, none of these approaches have made account for near point light sources that have varying effect depending on the distance. Thus, regarding indoor scenes, further accurate characterization of lighting is desired.

Recently, Debevec [10] uses a specular sphere as a light probe for measuring the incident illumination. Despite the accuracy of the estimated irradiance, such a sphere might have strong inter-reflections with other objects especially if they are close, which brings into question its validity in estimating near light sources. Also, Sato et al. [11] explicitly measure the 3D lighting distribution using stereo omnidirectional images that are captured with a fish-eye lens, and judge the positions of light sources by matching the highlights in the images. While this complete system is capable of representing the effects of point light sources, the irradiance is computed by interpolation on the bases of those accurate ones at cameras' projection centers and it is still difficult to achieve estimation of light sources that are in proximal scene. To our knowledge the most closely related work to our proposition in this respect is that by Powell et al. [12]. They present a technique for calibrating the light source geometry by matching highlights on three spheres. With this geometric matching method, it is possible to estimate the position of a light source although the radiant intensity of the light sources is not available.

In this paper we propose a novel method for estimating parameters of light sources, i.e., ambient light, directional light sources, and in particular near point light sources in- 


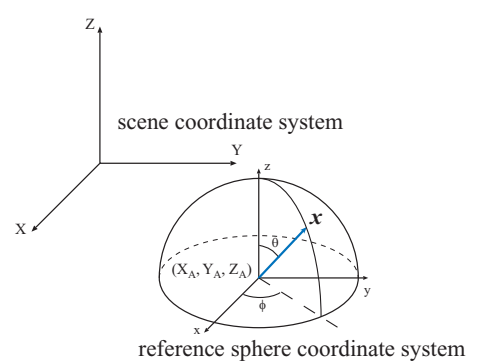

Figure 1. Coordinate systems.

cluding their radiant intensity. We employ a pair of reference spheres as a light probe and analyze gray level contours on the spherical surfaces so that 3D geometric and photometric properties of light sources can be characterized. Our key idea then is introducing the notion of difference sphere that we acquire by differencing two image regions of the reference spheres. We show that separate identification of multiple combined light sources is facilitated through the analysis of gray level contours on the difference sphere.

The sequel of the paper is composed as following. In Section 2 we define the lighting and reflectance model. While introducing the notion of difference sphere in Section 3 , we discuss the characteristics of it in Section 4. Section 5 provides the description of our algorithm of light source estimation followed by the experimental results in Section 6. Finally the paper is summarized in Section 7.

\section{Model Definitions}

Assumptions For our light source estimation we employ a pair of spheres with known size, which we call reference spheres, and assume Lambertian BRDFs. We place the spheres in a way that they do not occlude or cast shadows to each other. We also assume that the camera for capturing images and the reference spheres are accurately calibrated. We then deal with measured image irradiance which we in this paper refer to as image intensity ${ }^{1}$. We consider the mutual reflection between the spheres as minor and ignorable.

Coordinate systems As illustrated in Figure 1 we consider the scene coordinate system $(X, Y, Z)$ and the sphere coordinate system $(x, y, z)$. Given a reference sphere, $A$, we align each axis of the sphere coordinate system, $x_{A}, y_{A}$, and $z_{A}$, parallel to $X, Y$, and $Z$ axis of the scene coordinate system, respectively. We also utilize surface coordinate system $(\theta, \phi)$ in order to specify angular positions of spherical surface points.

Lighting environment As already mentioned, we deal with three types of light sources, i.e., near point light source, distant directional light source, and ambient light. In the fol-

1 In other words, we assume that the transformation function from the reflected intensity to the pixel gray value is spatially uniform and linear with zero bias so that the scale factor is equal to 1 .

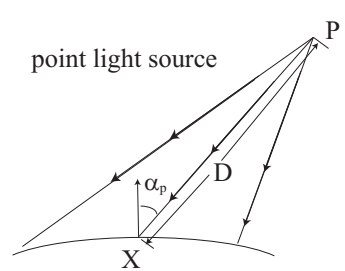

(a) Point light source

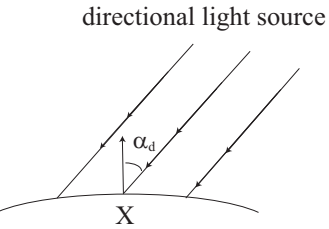

(b) Directional light source
Figure 2. Point light source and directional light source.

lowing descriptions we denote the reflectance coefficient of the reference sphere as $\eta$.

- Near point light source: As shown in Figure 2(a), at point $\boldsymbol{X}$, let $\alpha_{p}(\boldsymbol{X}, \boldsymbol{P})$ be an angle between the surface normal and the line from the light source. The reflected intensity, $I_{p}(\boldsymbol{X} ; \boldsymbol{P})$, is then given by

$$
I_{p}(\boldsymbol{X} ; \boldsymbol{P})=\eta L_{p} \max \left[\cos \alpha_{p}(\boldsymbol{X}, \boldsymbol{P}), 0\right] /(D(\boldsymbol{X}, \boldsymbol{P}))^{2},
$$

where $L_{p}$ denotes radiant intensity of the point light source, $\boldsymbol{P}$ the position of it, and $D(\boldsymbol{X}, \boldsymbol{P})$ the distance between the light source and $\boldsymbol{X}$.

- Directional light source: As shown in Figure 2(b), let $\alpha_{d}(\boldsymbol{X})$ be an angle between the surface normal at point $\boldsymbol{X}$ and the direction of the light source. With the radiant intensity of the directional light source, $L_{d}$, the reflected intensity, $I_{d}(\boldsymbol{X})$, is given by

$$
I_{d}(\boldsymbol{X})=\eta L_{d} \max \left[\cos \alpha_{d}(\boldsymbol{X}), 0\right] .
$$

- Ambient light: It provides constant light for a scene. We consider it as bias in this paper. If the scene were illuminated by ambient light $L_{a}$ alone, the reflected intensity $I_{a}$ of the reference sphere would simply be

$$
I_{a}=\eta L_{a} .
$$

Reflected Intensity Under the above described light sources the reflected intensity in the scene coordinate system can be modeled in general as

$$
I(\boldsymbol{X})=\sum_{i=1}^{s} I_{p}^{[i]}(\boldsymbol{X} ; \boldsymbol{P}[i])+\sum_{j=1}^{t} I_{d}^{[j]}(\boldsymbol{X})+I_{a},
$$

where $s$ and $t$ are (unknown) numbers of point light sources and directional light sources, respectively, $i$ and $j$ are indices of them, and $\boldsymbol{P}[i]$ is the 3D position of the $i$-th point light source in the scene coordinate system.

\section{Difference Sphere}

In order to facilitate separate identification of multiple combined light sources, in this section, we introduce the notion of difference sphere which we acquire by differencing two image regions of reference spheres.

Reflected intensity of single sphere Let us first consider a reference sphere with shading in the scene as single sphere ${ }^{2}$, 
$A$, and formulate the reflected intensity on the surface of the sphere in the sphere coordinate system.

Let $\boldsymbol{x}_{A}$ represent a point on the surface of single sphere $A$, and $\mathcal{F}_{A}\left(\boldsymbol{x}_{A}\right)$ the $3 \mathrm{D}$ position of $\boldsymbol{x}_{A}$ in the scene coordinate system. Representing the reflected intensity, $I(X)$, in equation (4) by $I_{A}\left(\boldsymbol{x}_{A}\right)$, and thus replacing $\boldsymbol{X}$ with $\mathcal{F}\left(\boldsymbol{x}_{A}\right)$, the constituents of it according to equations (1)-(3) are given by

$$
\begin{aligned}
I_{p}^{[i]}= & \eta_{A} L_{p}^{[i]} \max \left[\cos \alpha_{p}^{[i]}\left(\mathcal{F}_{A}\left(\boldsymbol{x}_{A}\right), \boldsymbol{P}[i]\right), 0\right] \\
& /\left(D\left(\mathcal{F}_{A}\left(\boldsymbol{x}_{A}\right), \boldsymbol{P}[i]\right)\right)^{2}, \\
I_{d}^{[j]}= & \eta_{A} L_{d}^{[j]} \max \left[\cos \alpha_{d}^{[j]}\left(\mathcal{F}_{A}\left(\boldsymbol{x}_{A}\right)\right), 0\right], \\
I_{a}= & \eta_{A} L_{a},
\end{aligned}
$$

where $\eta_{A}$ is the diffuse coefficient, $L_{p}^{[i]}$ and $L_{d}^{[j]}$ denote the radiant intensity of the $i$-th point light source and the $j$-th directional light source, respectively. Note that both $\cos \alpha_{p}$ and $\cos \alpha_{d}$ accompany an index of light source accordingly.

Reflected intensity of difference sphere We virtually generate a difference sphere, " $A-B$ ", from a pair of reference spheres, $A$ and $B$, that has the following properties.

- Geometry: The location and the radius of the difference sphere is inherited from those of single sphere $A$.

- Photometry: Let $I_{A}(\theta, \phi)$ and $I_{B}(\theta, \phi)$ denote the reflected intensities of single spheres, $A$ and $B$, respectively. The reflected intensity of difference sphere, $A-B$, is ${ }^{3}$

$$
I_{A-B}(\theta, \phi)=I_{A}(\theta, \phi)-I_{B}(\theta, \phi) .
$$

From equations (4) - (7) and (8) the reflected intensity, $I_{A-B}\left(x_{A-B}\right)$, at point $x_{A-B}$ on the surface of difference sphere $A-B$ is defined as follows:

$$
\begin{gathered}
I_{A-B}\left(\boldsymbol{x}_{A-B}\right)=I_{A}\left(\boldsymbol{x}_{A}\right)-I_{B}\left(\boldsymbol{x}_{B}\right) \\
=\sum_{i=1}^{s} I_{p}^{[i]}\left(\mathcal{F}_{A}\left(\boldsymbol{x}_{A}\right) ; \boldsymbol{P}[i]\right)-\sum_{i=1}^{s} I_{p}^{[i]}\left(\mathcal{F}_{B}\left(\boldsymbol{x}_{B}\right) ; \boldsymbol{P}[i]\right)
\end{gathered}
$$

where surface coordinates of $\boldsymbol{x}_{A-B}, \boldsymbol{x}_{A}$, and $\boldsymbol{x}_{B}$ on their corresponding spheres are all equivalent. Equation (9) is due to the fact that the differencing operation in equation (8) eliminates lighting effects caused by all the directional light sources and ambient light. That is, the illumination on a difference sphere is caused only by point light sources.

Further, since the differencing operation generally gives rise to negative intensities as well as positive ones, we could interpret the surface intensities of a difference sphere as if they were independently generated by positive and negative point light sources. Thus, a difference sphere is virtually illuminated by positive and negative point light sources, each of which introduces positive and negative intensities.

Figure 3 illustrates a difference sphere that is generated by a pair of single spheres, and show the positive and the negative intensities. In the subsequent analysis of surface

2 We use the term, single sphere, interchangeably with reference sphere to explicitly distinguish it from difference sphere.

3 Note that in the shading analysis that is described later, $I_{A-B}(\theta, \phi)$ becomes undefined for those $(\theta, \phi)$ where the corresponding surface points on single spheres $A$ or $B$ cannot be observed by a camera.

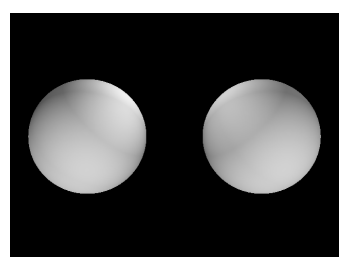

(a) Single spheres

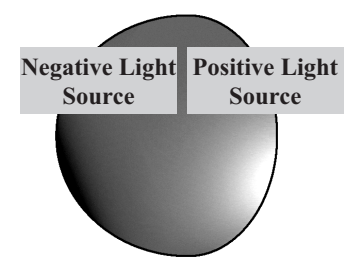

(b) Difference sphere
Figure 3. Single spheres and difference sphere. The gray-level of (b) shows the intensity of the difference sphere; the bright/dark area reflects positive/negative values. The intensities are emphasized for display reason.

intensities for estimating point light sources, negative intensities can be treated just equally as positive intensities without a loss of generality. Moreover, it turns out that we only need to analyze either positive intensities or negative ones for estimating the point light sources since the influence of the light sources basically appear in both of them. However, note that the acquired lighting parameters in case of analyzing negative intensities should be interpreted in the coordinate system whose origin is at the center of single sphere $B$, instead of sphere $A$. Although it does not really matter which one to choose in the example of Figure 3, it is in general sensible to choose the intensities that represent larger amount of radiant energy.

\section{Characteristics of Single and Difference Spheres}

Let us investigate the characteristics of the sphere surface while separating it into three categories ${ }^{4}$ depending on the types of illuminating light sources (see Figure 4). They are,

$S$-surface: The surface illuminated by a single light source (and the ambient light). In particular, we call $S$-surface that is illuminated by a single point light source $S_{p}$-surface and that by a single directional light source $S_{d}$-surface.

$M$-surface: The surface illuminated by multiple point and/or directional light sources.

$A$-surface: The surface illuminated by ambient light alone.

Now, we put our focus on the characteristics of $S$-surface in order to estimate parameters of light sources. We will see that the $S$-surface has such features as described below.

Feature 1: A set of points on $S$-surface with identical intensities form an arc on a plane in 3D scene. We call the plane feature plane.

Feature 2: The surface normal of the feature plane denotes the direction of the point/directional light source which illuminates the $S$-surface.

Feature 3: An intensity ratio of a group of feature planes in

4 Completely dark surface due to shadow is set aside. 

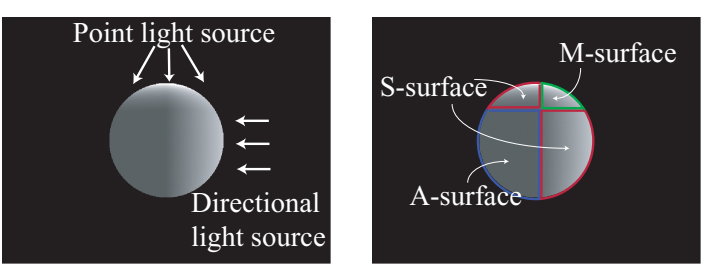

Figure 4. Surface classification. A reference sphere under light sources (left) and divided surfaces (right).

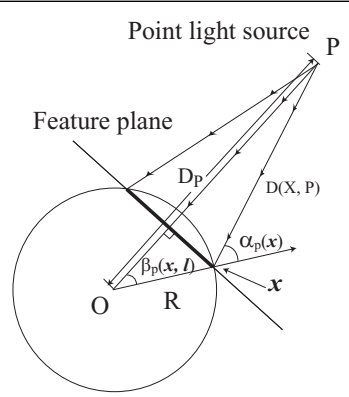

Figure 5. Relationship between a single point light source and a reference sphere.

an identical $S$-surface characterizes the radiant intensity of the light source.

In the remainder of the section, we explain the above features in detail. Preceded by the analyses in the cases of a single point light source and a single directional light source which show the mechanisms of our light source estimation, we derive how the difference sphere simplifies the procedure of light source estimation. Note that we additionally define the following notations.

$\boldsymbol{O}=\left(O_{X}, O_{Y}, O_{Z}\right):$ The center of a reference sphere.

$\boldsymbol{P}=\left(P_{X}, P_{Y}, P_{Z}\right):$ The light source position.

$\boldsymbol{l}=\left(l_{X}, l_{Y}, l_{Z}\right)$ : The direction of the light source.

$R$ : The radius of a reference sphere.

\subsection{Characteristics of $S_{p}$-surface}

The bold line in Figure 5 illustrates a set of surface points whose intensities are equal to $I(\boldsymbol{x})$. As is obvious from the figure, such points form an arc on the feature plane (Feature 1). In Figure 5, we can easily prove that the surface normal of the feature plane coincides with the direction of the point light source. Then, the feature plane is defined by

$$
\begin{aligned}
& l_{X}\left(X-\left(O_{X}-l_{X} R \cos \beta_{p}(\boldsymbol{x}, \boldsymbol{l})\right)\right) \\
+\quad & l_{Y}\left(Y-\left(O_{Y}-l_{Y} R \cos \beta_{p}(\boldsymbol{x}, \boldsymbol{l})\right)\right) \\
+\quad & l_{Z}\left(Z-\left(O_{Z}-l_{Z} R \cos \beta_{p}(\boldsymbol{x}, \boldsymbol{l})\right)\right)=0
\end{aligned}
$$

where $\left(l_{X}, l_{Y}, l_{Z}\right)=\left(O_{X}-P_{X}, O_{Y}-P_{Y}, O_{Z}-P_{Z}\right)$, and $\beta_{p}(\boldsymbol{x}, \boldsymbol{l})$ denotes the angle between $\boldsymbol{l}$ and the line connecting $\boldsymbol{O}$ and $\boldsymbol{x}$ (Feature 2). Note that equation (10) tells that all feature planes that is defined in an $S_{p}$-surface are parallel to each

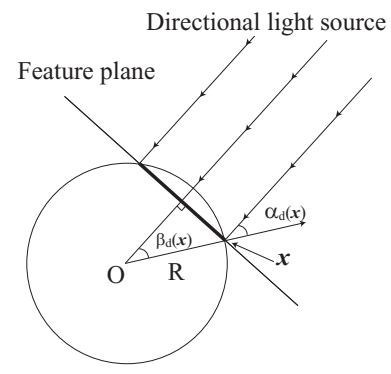

Figure 6. Relationship between a single directional light source and a reference sphere.

other. Furthermore, a group of feature planes with different intensities characterizes the radiance of the point light source illuminating the $S_{p}$-surface. Since the intensity of the group of feature planes varies according to $\beta_{p}(\boldsymbol{x}, \boldsymbol{l})$, we describe the intensity with $\beta_{p}(\boldsymbol{x}, \boldsymbol{l})$ instead of $\alpha_{p}(\mathcal{F}(\boldsymbol{x}), \boldsymbol{P})$.

The reflected intensity, $I(\boldsymbol{x})$, at $\boldsymbol{x}$ on a feature plane is

$$
I(\boldsymbol{x})=\eta L_{p} \cos \alpha_{p}(\mathcal{F}(\boldsymbol{x}), \boldsymbol{P}) /(D(\mathcal{F}(\boldsymbol{x}), \boldsymbol{P}))^{2}+\eta L_{a},
$$

where $\cos \alpha_{p}(\mathcal{F}(\boldsymbol{x}), \boldsymbol{P})$ and $D(\mathcal{F}(\boldsymbol{x}), \boldsymbol{P})$ can be formulated as

$$
\begin{aligned}
\cos \alpha_{p}(\mathcal{F}(\boldsymbol{x}), \boldsymbol{P}) & =\frac{D_{P}^{2}-R^{2}-(D(\mathcal{F}(\boldsymbol{x}), \boldsymbol{P}))^{2}}{2 R D(\mathcal{F}(\boldsymbol{x}), \boldsymbol{P})}, \\
D(\mathcal{F}(\boldsymbol{x}), \boldsymbol{P}) & =\sqrt{D_{P}^{2}+R^{2}-2 R D_{P} \cos \beta_{p}(\boldsymbol{x}, \boldsymbol{l})}
\end{aligned}
$$

Notice $D_{P}$ denotes the distance between $\boldsymbol{O}$ and $\boldsymbol{P}$. By substituting the above equations into equation (11), we have

$$
I(\boldsymbol{x})=\eta L_{p} \frac{D_{P} \cos \beta_{p}(\boldsymbol{x}, \boldsymbol{l})-R}{\left(D_{P}^{2}+R^{2}-2 R D_{P} \cos \beta_{p}(\boldsymbol{x}, \boldsymbol{l})\right)^{\frac{3}{2}}}+\eta L_{a} .
$$

Equation (14) indicates that the reflected intensity depends on unknown $L_{p}, L_{a}$, and $D_{P}$. However, the ambient light term can be eliminated by subtracting the intensities at points on any two feature planes. Besides, the lighting effect of the point light source due to $L_{p}$ can also be canceled by selecting yet another point on any other feature plane and computing the intensity ratio,

$$
\frac{I\left(x_{1}\right)-I\left(x_{2}\right)}{I\left(x_{1}\right)-I\left(x_{3}\right)}=\frac{I^{\prime}\left(x_{1}\right)-I^{\prime}\left(x_{2}\right)}{I^{\prime}\left(x_{1}\right)-I^{\prime}\left(x_{3}\right)}
$$

where

$$
I^{\prime}(\boldsymbol{x})=\frac{D_{P} \cos \beta_{p}(\boldsymbol{x}, \boldsymbol{l})-R}{\left(D_{P}^{2}+R^{2}-2 R D_{P} \cos \beta_{p}(\boldsymbol{x}, \boldsymbol{l})\right)^{\frac{3}{2}}} .
$$

Solving equation (15), we can theoretically obtain $D_{P}$, and thereby $L_{p}$ and $L_{a}$ from equation (14) (Feature 3). Nevertheless, the solution to $D_{P}$ is not indeed straightforward, which indicates the difficulty in estimating the point light sources solely by a single sphere.

\subsection{Characteristics of $S_{d}$-surface}

In Figure 6 we can prove Feature 1 and Feature 2 of $S_{d^{-}}$ surface in the same way as in the case with $S_{p}$-surface. The 
feature plane can be described as

$$
\begin{aligned}
& l_{X}\left(X-\left(O_{X}-l_{X} R \cos \beta_{d}(\boldsymbol{x})\right)\right) \\
+\quad & l_{Y}\left(Y-\left(O_{Y}-l_{Y} R \cos \beta_{d}(\boldsymbol{x})\right)\right) \\
+\quad & l_{Z}\left(Z-\left(O_{Z}-l_{Z} R \cos \beta_{d}(\boldsymbol{x})\right)\right)=0
\end{aligned}
$$

(Feature2).

The reflected intensity, $I(\boldsymbol{x})$, at $\boldsymbol{x}$ on an $S_{d}$-surface is then

$$
I(\boldsymbol{x})=\eta L_{d} \cos \alpha_{d}(\boldsymbol{x})+\eta L_{a} .
$$

We rewrite this equation with $\beta_{d}(\boldsymbol{x})$ to

$$
I(\boldsymbol{x})=\eta L_{d} \cos \beta_{d}(\boldsymbol{x})+\eta L_{a},
$$

where $\beta_{d}(\boldsymbol{x})=\alpha_{d}(\boldsymbol{x})$. Selecting points on any three independent feature planes, we have the intensity ratio

$$
\frac{I\left(x_{1}\right)-I\left(x_{2}\right)}{I\left(x_{1}\right)-I\left(x_{3}\right)}=\frac{I^{\prime}\left(x_{1}\right)-I^{\prime}\left(x_{2}\right)}{I^{\prime}\left(x_{1}\right)-I^{\prime}\left(x_{3}\right)}
$$

where $I^{\prime}(\boldsymbol{x})=\cos \beta_{d}(\boldsymbol{x})$.

To summarize the analysis on $S$-surface, if the intensity ratio is given by equation (18), we can deduce that the $S$ surface is an $S_{d}$-surface and thus can obtain $L_{d}$ and $L_{a}$ from equation (17). Otherwise the $S$-surface is an $S_{p}$-surface, and we need to go through equations (15) and then (14) in order to obtain the lighting parameters, which is a hard problem as we just saw in the previous section.

\subsection{Characteristics of Difference Sphere}

$S$-surface of a difference sphere has similar characteristics as does $S_{p}$-surface of a single sphere, except that the factor of ambient light is precluded. That is, intensity $I(\boldsymbol{x})$ at $\boldsymbol{x}$ on a difference sphere is

$$
I(\boldsymbol{x})=\eta L_{p}^{*} \cos \alpha_{p}(\mathcal{F}(\boldsymbol{x}), \boldsymbol{P}) /(D(\mathcal{F}(\boldsymbol{x}), \boldsymbol{P}))^{2},
$$

where $L_{p}^{*}$ denotes the radiant intensity of a positive or negative point light source. Thus the intensity ratio is given by

$$
\frac{I\left(x_{1}\right)}{I\left(x_{2}\right)}=\frac{I^{\prime}\left(x_{1}\right)}{I^{\prime}\left(x_{2}\right)}
$$

where

$$
I^{\prime}(\boldsymbol{x})=\frac{D_{P} \cos \beta_{p}(\boldsymbol{x}, \boldsymbol{l})-R}{\left(D_{P}^{2}+R^{2}-2 R D_{P} \cos \beta_{p}(\boldsymbol{x}, \boldsymbol{l})\right)^{\frac{3}{2}}} .
$$

Solving equation (20) analogously, we can obtain $D_{p}$, and then $L_{p}^{*}$ from equation (19). It can be seen that a more practical solution is allowed in the case with difference sphere and it is much simpler than the case of solving equation (15) with single sphere.

\section{Algorithm of Light Source Estimation}

Based on the above discussions we propose a twofold algorithm for light source estimation as following:

step 0 Capture an image.

step 1 Generate an image of a difference sphere

step 1-1 Estimate parameters of point light sources step 2 Update the input image by eliminating the lighting effects that is due to the estimated point light sources.

step 2-1 Estimate parameters of directional light sources and ambient light.

In each sub-step (1-1, 2-1), we estimate the parameters of light sources by an iterative operation. That is, we eliminate the effects of light source candidates one after another from the input image, and verify them by analyzing the residual in the image (Figure 7). The procedure is:

i. Convert each image region of the sphere to contour representation (see Section 5.1).

ii. Estimate parameters of light source candidates (see Section 5.2).

$1^{\circ}$ Divide the contour lines into contour segments

$2^{\circ}$ Extract candidates of $S$-surfaces.

$3^{\circ}$ Analyze each candidate of $S$-surface and estimate a corresponding light source candidate.

iii. Verify each of the light source candidates (see Section 5.3).

$1^{\circ}$ Generate an image by eliminating the effect of a light source candidate from the input image.

$2^{\circ}$ Analyze the residual intensity.

- If the surface has a uniform value, we regard the light source candidates that have been employed for the generation of the input image as correct and terminate the procedure.

- If there exists a surface with negative values (negative surface), the light source candidate is judged to be incorrect.

- Otherwise, update the input image to the image that is generated by $\mathbf{i i i - 1} 1^{\circ}$ and go to $\mathbf{i}$.

We now describe the details of the processes in sub-steps $\mathbf{i}$ to iii.

\subsection{Region Representation with Contour Lines}

The contour representation can be described as a geographic map where the intensity levels of pixels are regarded as height at corresponding locations [13]. After obtaining the contour representation, we use it for extracting $S$-surfaces in the input image. Since the contour representation reflects geometric characteristics of shading of objects while being robust against local noise, we consider that it is more suitable for extraction and analysis of $S$-surfaces than an ordinary image representation by an array of pixel values.

\subsection{Estimation of Light Source Candidates' Pa- rameters}

As the surface of a sphere is illuminated by multiple light sources, the contour naturally consists of multiple segments, rather than looking like a simple arc. Thus, we first divide every single contour line into contour segments in such a way that each segment represents an arc. The contour segmentation is an important process because it is the very clue 


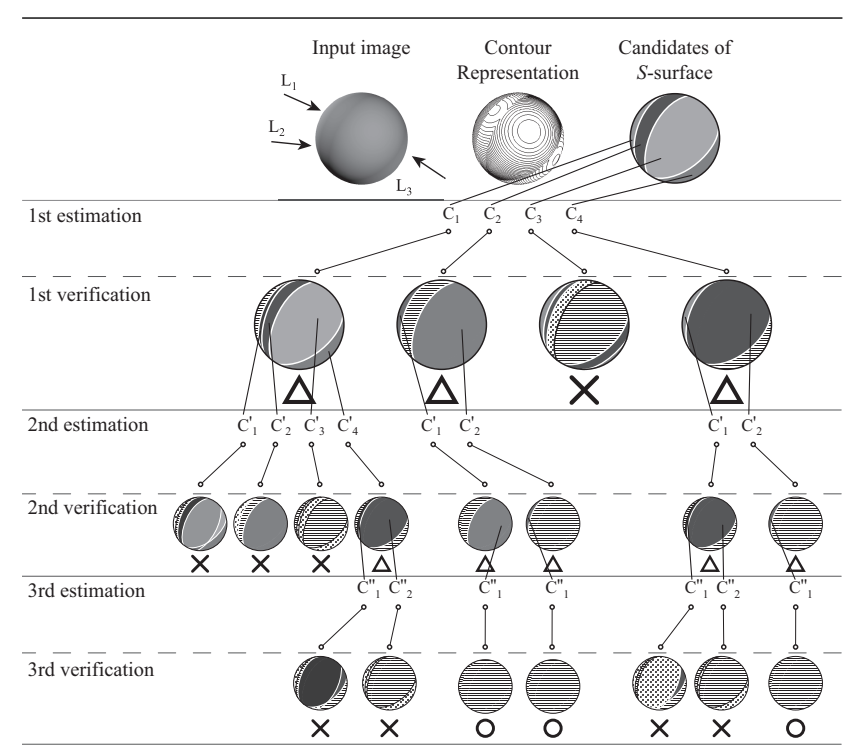

$\square$ completely eliminated surface negative surface

Figure 7. An example of an estimation flow. ' $L$ ' denotes the light sources, and ' $C$ ' denotes $S$ surface candidates. $\bigcirc$ and $\times$ signify correct and incorrect estimations, respectively, and $\Delta$ an intermediate estimation.

with physical meanings for extracting an $S$-surface as a unit which we analyze for estimating the corresponding lighting source. By adapting the feature of contour segment representation of $S$-surface, we rewrite Feature 1 to Feature 1'. Namely,

Feature 1': A contour segment on an $S$-surface forms an arc on a plane in 3D scene. We call the plane feature plane.

We then estimate the light source parameters as following:

$1^{\circ}$ We divide the contour lines into contour segments based on Feature 1'.

$2^{\circ}$ By grouping the contour segments which indicate feature planes whose surface normals are in identical directions, we extract $S$-surface(s).

$3^{\circ}$ We estimate the parameters of the light sources according to Feature 2 while calculating the intensity ratio to determine the radiant intensity by Feature 3.

\subsection{Verification of Estimated Light Source Candi- dates}

We verify the estimated light source candidates by analyzing images that are generated by eliminating their possible effects from the input image. That is, if the residual has negative values ${ }^{5}$, we can determine that the estimation is not correct.

Suppose a reference sphere that is illuminated by three directional light sources (Figure 7). While there are four candidates of $S$-surface in the input image, correct

5 In step 1, the residual should be zero. In step 2, the residual can also take a uniform value, which is regarded as the effect of ambient light.
$S$-surfaces are $\mathrm{C}_{2}$ and $\mathrm{C}_{4}$, and the others are $M$-surfaces. Although it is not possible to identify $S$-surfaces among the candidates only by the iso-intensity contours, we can identify an $M$-surface by analyzing the residual that is generated by eliminating the effects of the light source candidates. That is, we can identify that $\mathrm{C}_{3}$ is an $M$-surface since the negative surface appears in the third figure from the left in the first verification, which is generated by eliminating the effect of the light source candidate from $\mathrm{C}_{3}$. Continuing the procedure iteratively with the updated images, we find that three paths give the correct estimations. As these paths allow estimations of identical parameters of the same light sources, the lighting environment is correctly estimated.

For general lighting setting, as long as at least one correct $S$-surface exists, we can estimate the corresponding light source and continue the procedure by eliminating its effect from the input image accordingly.

\section{Experimental Results}

We demonstrate the effectiveness of our algorithm with a CG image and a real image. The CG image is rendered with OpenGL while Gaussian noise with a variance of 6.55 is added to it.

\subsection{Results: CG Image}

Figure 8 exemplifies some results including the contour analysis on difference sphere. The input spheres are set to be illuminated by a point light source, two directional light sources, and ambient light. Each figure shows:

(a) Input image.

(b) Difference sphere generated from (a).

(c) Grouped contour segments and contour lines of the region that has positive values.

(d) Grouped contour segments and contour lines of the region that has negative values.

(e) Intermediate image - generated by eliminating the lighting effect by the estimated point light source from the input image.

(f) Grouped contour segments and contour lines of (e).

(g) Result of elimination of lighting effects.

The intermediate image (e) shows the situation where the lighting effect by the point light source is eliminated and the remaining effect by directional light sources and ambient light is present. We then consider the image as a new input and estimate the parameters of light sources with the reference spheres which we regard as two reference spheres. (f) shows that each single sphere reflects one directional light source, respectively, whereas $(\mathrm{g})$ is a result of eliminating the effect by the light sources.

Table 1 shows the estimated parameters as the result. LS1 is best estimated whereas the accuracy tends to relatively decline as the estimation proceeds due to accumulation of errors. However, it can be seen that the overall performance is quite reasonable as the first trial of estimating both the positions and the intensities of light sources. 


\begin{tabular}{|l|l||c|c|}
\hline & parameter & true & estimated \\
\hline LS1 & type & point & point \\
& intensity & 127.5 & 120.4 \\
& position & $(0.0,-2.0,-1.0)$ & $(-0.006,-1.99,-1.01)$ \\
\hline LS2 & type & directional & directional \\
& intensity & 76.5 & 77.67 \\
& direction & $(45.9,14.0)$ & $(47.2,14.6)$ \\
\hline LS3 & type & directional & directional \\
& intensity & 76.5 & 93.77 \\
& direction & $(73.3,0.0)$ & $(70.6,-8.9)$ \\
\hline Amb. & intensity & 76.0 & 68.16 \\
\hline
\end{tabular}

Table 1. Estimated parameters of lighting environment in CG image. The estimation is for light source 1-3 and ambient light source (LS1LS3 and Amb.). The direction of directional light source is represented in $(\theta, \phi)$.

\subsection{Results: Real Image}

Figure 9 shows results of lighting environment estimation for a real scene. Figure 9(a) shows an input image of a real scene which includes a point light source and two directional light sources, one roughly from the upper-right of the viewing direction and the other from the opposite side. Two reference spheres are placed on mounts whereas the point light source is located in between them and hidden by the frontal sphere. Images in Figure 9(b), (c), and (d) are generated by illuminating a CG teapot by each of the estimated light source, respectively, whereas (e) is with all the three light sources. Figure 9(f) shows a synthesized image by adding the virtual object and the shadows in (e) into the real scene. The point light source is now visible since the spheres have been removed while two little real dolls are placed for comparison. As the point light source is not completely isotropic and illuminates only the upper hemisphere from a certain height, it may appear odd that the floor remains dark. Apart from that it illustrates that a CG object is added naturally with the real lighting environment.

Furthermore, Figure 9(g) shows virtual spheres in the estimated lighting environment, and (h) shows the difference between (a) and ( $\mathrm{g}$ ). It illustrates that in the estimated lighting environment the virtual spheres are illuminated almost equivalently as in the input lighting environment. The errors that appear around the larger sphere due to calibration errors.

Finally, we show the estimated light source positions and the intensities of the real scene (Table 2) under the following scene coordinate system:

Origin: center of the bottom of the virtual teapot,

$\mathbf{X}$-axis: towards the right of the horizontal direction of the image,

Y-axis: along the depth direction of the plane,

Z-axis: orthogonal to the XY-plane.

\section{Discussions}

We have presented a novel technique for lighting environment estimation which allows us to estimate parameters of point light sources as well as directional light sources and ambient light. We employ a contour representation of an

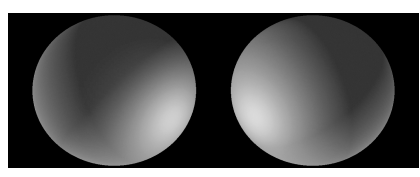

(a) Input image

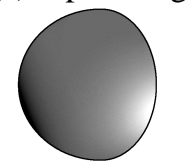

(b) Difference sphere

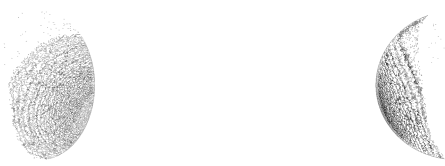

(c) Grouped contour segments and contour lines of the positive light source (d) Grouped contour segments and contour lines the negative light source

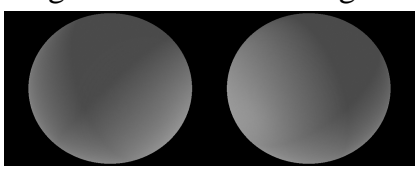

(e) Intermediate image

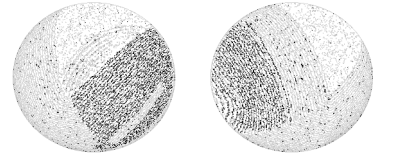

(f) Grouped contour segments and contour lines

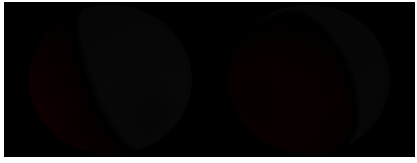

(g) Result of elimination of lighting effects

Figure 8. The procedures of light source estimation.

image for analyzing shading of a pair of reference spheres and characterize 3D geometric and photometric properties of the light source. In particular we have proposed the difference sphere which enables us to estimate parameters of the point light sources by eliminating the lighting effects of ambient light and directional light sources. In order to show the theoretical availability, we have demonstrated the effectiveness by applying our method to CG images generated with a point light source, two directional light sources, and ambient light.

As a future work, first we would like to examine our method in a further complex lighting environment since the current algorithm has naturally limitations even though we have described our algorithm as a general framework. For instance, notwithstanding the verification of $S$-surfaces, it will be difficult to deal with the cases with a very large number of light sources which make the identification of $S$-surface(s) complicated. In order to properly handle the situation, however, it should be useful to consider critical points for region segmentation, as discussed in [14]. An- 


\begin{tabular}{|l|l||c|}
\hline & parameter & estimated \\
\hline LS1 & type & point \\
& intensity & 143.0 \\
& position & $(1.16,-25.9,2.35)$ \\
\hline LS2 & type & directional \\
& intensity & 143.3 \\
& direction & $(61.31,122.40)$ \\
\hline LS3 & type & directional \\
& intensity & 103.0 \\
& direction & $(65.80,-54.47)$ \\
\hline
\end{tabular}

Table 2. Estimated parameters of lighting environment in real scene. The estimation is for light source 1-3 (LS1-LS3). The direction of directional light source is represented in $(\theta, \phi)$.

other aspect that we wish to investigate is the availability of Lambertian reflectance model for estimation of complex lighting environment. Since the Lambertian reflectance model performs low-pass filtering of the lighting environment $[15,16,17]$, the problem may be ill-posed or numerically ill-conditioned. For the problem to be alleviated, we need to consider the configuration of reference spheres for effectively generating the difference sphere although our algorithm should work once the $S$-surfaces are identified, even if the lighting environment is rather complex. Also, the other direction of extensions will be to model other light sources that have size and range, for estimating natural illumination.

Acknowledgements This work is supported by Grant-inAid for Scientific Research of the Ministry of Education, Culture, Sports, Science and Technology of Japan under the contraction of 13224051 .

\section{References}

[1] B.K.P. Horn: Image intensity understanding. Artificial Intelligence, Vol.8, pp. 201-231, 1977.

[2] A. P. Pentland: Finding the illumination direction. Journal of Optical Society of America, Vol. 72, No. 4, pp. 448-455, 1982.

[3] Q. Zheng, R. Chellappa: Estimation of illuminant direction, albedo, and shape from shading. IEEE Trans. PAMI, vol. 13, no. 7, pp. 680702, 1991.

[4] D. R. Hougen and N. Ahuja: Estimation of the light source distribution and its use in integrated shape recovery from stereo and shading. 4th ICCV, pp. 148-155, 1993.

[5] S. R. Marschner and D. P. Greenberg: Inverse lighting for photography. In Fifth Color Imaging Conference, pp. 262-265, 1997.

[6] Y. Yang and A. Yuille: Sources from shading. In Proc. IEEE Conference on Computer Vision and Pattern Recognition 99, pp. 534-539, 1991.

[7] Y. Zhang and Y. Yang: Multiple illuminant direction detection with application to image synthesis. IEEE Trans. PAMI, Vol.23, pp. 915920, 2001

[8] W. Zhou and C. Kambhamettu: Estimation of illuminant direction and intensity of multiple light sources. 7th ECCV, pp. 206-220, 2002.

[9] A. Maki: Estimation of Illuminant Direction and Surface Reconstruction by Geotensity Constraint. Pattern Recognition Letters, Vol. 21:13-14, pp. 1115-1123, 2000.

[10] P. Debevec: Rendering synthetic objects into real scenes: Bridging traditional and image-based graphics with global illumination and high dynamic range photography. In Proc. ACM SIGGRAPH, pp. 189-198, 1998.

[11] I. Sato, Y. Sato, and K. Ikeuchi: Acquiring a radiance distribution to superimpose virtual objects onto a real scene. IEEE Trans. VCG, vol. 5, no. 1, pp. 1-12, 1999 .

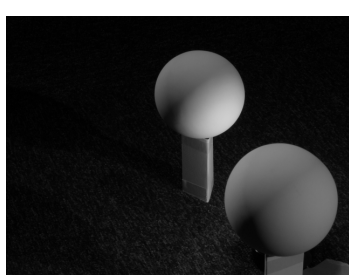

(a) Input image

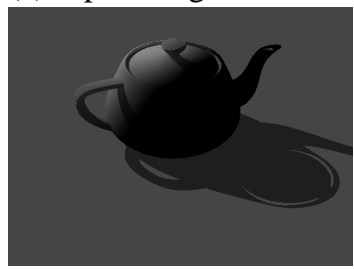

(c) Directional light source 1

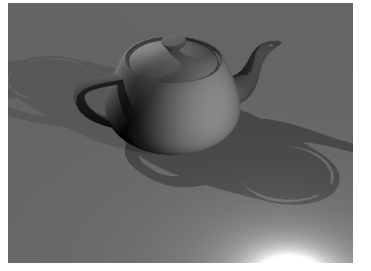

(e) Estimated light sources

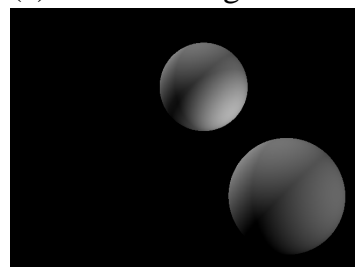

(g) Virtual spheres in the estimated lighting environment

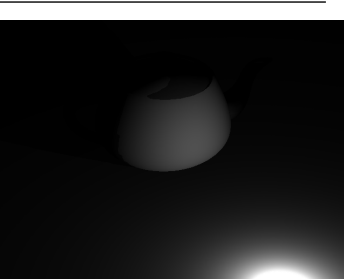

(b) Point light source

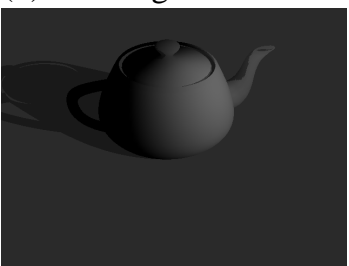

(d) Directional light source 2

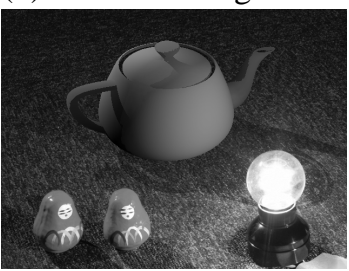

(f) Synthesized image
Figure 9. The experiment of adding virtual object in a real scene. Images in (b), (c), and (d) are generated by illuminating a virtual object by each estimated light source, respectively. (e) is generated with the above three light sources, (f) is a synthesized image in the real scene. $(g)$ shows virtual spheres in the estimated lighting environment, and $(h)$ the difference between (a) and $(g)$.

[12] M. W. Powell, S. Sarkar, and D. Goldgof: A simple strategy for calibrating the geometry of light sources. IEEE Trans. PAMI Vol.23, pp. 1022-1027, 2001

[13] T. Asano and S. Kimura: Contour representation of an image with applications. IPSJ Signotes Algorithms, No.058-009, pp. 65-70, 1997.

[14] Y. Wang and D. Samaras: Estimation of multiple illuminants from a single image of arbitrary known geometry. 7th ECCV, pp. 272-288, 2002.

[15] R. Ramamoorthi and P. Hanrahan: A signal-processing framework for inverse rendering. In Proc. ACM SIGGRAPH, pp. 117-128, 2001.

[16] R. Basri and D. W. Jacobs: Lambertian reflectance and linear subspaces. IEEE Trans. PAMI, Vol.25, No. 2, pp. 218-233, 2003.

[17] P. Nillius and J.-O. Eklundh: Low-dimensional representation of shaded surfaces under varying illumination. In Proc. IEEE Conference on Computer Vision and Pattern Recognition, pp. II-185-192, 2003. 